\title{
Local antimicrobials in addition to scaling and root planing provide statistically significant but not clinically important benefit
}

\author{
Abstracted from \\ Matesanz-Pérez P, García-Gargallo M, Figuero E, Bascones-Martínez A, Sanz M, Herrera D. \\ A systematic review on the effects of local antimicrobials as adjuncts to subgingival debridement, \\ compared with subgingival debridement alone, in the treatment of chronic periodontitis. J Clin Periodontol 2013 ; 40: $227-241$. \\ Address for correspondence: David Herrera, Facultad de Odontología Universidad Complutense Plaza Ramón y Cajal s/n, \\ Ciudad Universitaria 28040 Madrid, Spain. E-mail: davidher@odon.ucm.es
}

\section{Question: In treatment of chronic periodontitis does the use of local antimicrobial agents as an adjunct to subgingival debridement improve outcomes?}

Data sources The databases Medline, Embase and the Cochrane Oral Health Group Trials Register were searched. Handsearching of the Journal of Periodontology, Journal of Clinical Periodontology and Journal of Periodontal Research was also carried out.

Study selection Randomised controlled trials published in English were included.

Data extraction and synthesis Risk of bias and quality assessment were conducted following Cochrane recommendations with data being extracted independently in duplicate. Because of the nature of the available data, qualitative summary is presented with meta-analysis conducted where appropriate.

Results The overall effect of the subgingival application of antimicrobials was statistically significant $(p=0.000)$ for both changes in probing pocket depth (PPD) and clinical attachment level (CAL), with a weighted mean difference (WMD) of -0.407 and $-0.310 \mathrm{~mm}$ respectively. No significant differences occurred for changes in bleeding on probing (BOP) and plaque index (PII). Subgingival application of tetracycline fibres, sustained released doxycycline and minocycline demonstrated a significant benefit in PPD reduction (WMD between 0.5 and $0.7 \mathrm{~mm}$ ). The rest of the tested outcomes demonstrated a high heterogeneity. The local application of chlorhexidine and metronidazole showed a minimal effect when compared with placebo (WMD between 0.1 and $0.4 \mathrm{~mm}$ ).

Conclusions In conclusion, the scientific evidence supports the adjunctive use of local antimicrobials to SRP in deep or recurrent periodontal sites, mostly when the vehicle has shown pharmacodynamic properties assuring the sustained release of the antimicrobial. This evidence must be interpreted with caution, as the reported data were highly heterogeneous and most of the selected studies were categorised with a high degree of bias.

\section{Commentary}

Numerous clinical studies have shown that thorough mechanical debridement (ie scaling and root planing) is quite effective in the management of most cases of chronic periodontitis. The principle behind this is that disruption of the plaque biofilm and removal of local factors that harbour periodontal pathogens results in a reduction in periodontal inflammation and pocket depth - facilitating home care by the patient. However, subgingival debridement is a time-consuming and difficult procedure, highly dependent upon the skill of the clinician. ${ }^{1,2}$ Even when meticulously performed it does not necessarily eliminate pathogenic bacteria from the subgingival site. This is the basis for the rationale that the adjunctive use of antimicrobials could benefit patients with aggressive or refractory disease as well as those with local sites of disease. Local antimicrobials are particularly appealing as they do not carry the same risk of patient sensitivity and bacterial resistance associated with systemic antibiotics. For over 20 years, various classes of drugs and systems of drug delivery have been investigated to complement mechanical debridement.

The systematic review by Matesanz-Pérez et al. asks the question 'what are the effects of local antimicrobials as adjuncts to subgingival debridement, compared with subgingival debridement alone, in chronic periodontitis patients, in terms of clinical outcomes?'. The review was well conducted, with few limitations. A thorough search of the literature found 52 studies, of which 41 were included in meta-analyses. Despite the fact the search was limited to randomised controlled trials, only two studies were judged to be at low risk of bias. Eight of the 52 studies reported adequate randomisation methodology; of these only two used adequate allocation concealment. Blinding of the evaluators in clinical trials is essential to prevent over- or underestimation of treatment effects; over one third of the studies in this review did not report on blinding. An additional, and not insignificant, potential source of bias is the funding of studies. Forty- six of the 52 trials either did not state the source of funding, or were funded by industry.

While there was a statistical trend that favoured the use of adjunctive antimicrobials, it is important to dissect this information out. For example, the overall summary scores in the meta-analyses may have been statistically significant for some drugs, but are not likely clinically important, ${ }^{3}$ as the addition of antimicrobials provided an additional $0.3 \mathrm{~mm}$ increase in attachment level or $0.4 \mathrm{~mm}$ decrease in probing depth over scaling and root planing alone. There was no statistical (or clinical) advantage for the use of metronidazole or chlorhexidine varnish or gel. When separating out the split-mouth 


\section{PERIODONTOLOGY}

trials from the parallel trials, differences for the other drugs, while still statistically significant, were much lower. This is not surprising, since, even though the drugs are being used in a localised area, it is not clear that there is not a carry-over effect. Tetracycline - either as fibres or doxycycline gel - had the most promising (and precise) results, both in the short, medium and long term. Nonetheless, the overall results were still not clinically important.

This review points out several weaknesses in current research. Of the studies conducted in the last ten years included in this review, only one followed the CONSORT guidelines for study design - ensuring adequate sequence generation, allocation concealment, minimal loss-to-follow-up and appropriate blinding. It is important for funding bodies and journal editors to set high standards for clinical research so that, as clinicians, we can be comfortable that the scientific evidence upon which we base our clinical decisions is sound and meaningful.

\section{Debora Matthews}

Department of Dental Clinical Sciences, Dalhousie University, Halifax, Nova Scotia, Canada

1. Badersten A, Nilveus R, Egelberg J. Effect of nonsurgical periodontal therapy. III. Single versus repeated instrumentation. / Clin Periodontol 1984; 11: 114-124.

2. Badersten A, Nilveus R, Egelberg J. Effect of nonsurgical periodontal therapy (IV) Operator variability. J Clin Periodontol 1985; 12: 190-200.

3. Greenstein G. and Lamster I. Efficacy of periodontal therapy: statistical versus clinical significance. J Periodontol 2000; 71: 657-662.

Evidence-Based Dentistry (2013) 14, 87-88. doi:10.1038/sj.ebd.6400955 